\title{
Clinical Dilemmas in the Management of a Monocular Patient with Down's Syndrome, Dementia, Advanced Keratoconus, and a Mature Cataract
}

\author{
${ }^{1}$ Hala Ali, ${ }^{2}$ Mayank A Nanavaty
}

\begin{abstract}
There are reports on the successful management of mild to moderate keratoconus in patients with Down's syndrome. However, when Down's syndrome is coexistent with Dementia, poor general health, and very advanced keratoconus in only one eye, then clinicians may face challenges at all stages from diagnosis, treatment, and follow-up. Our case is one such example of a combination of Down's syndrome, Dementia, poor general health, and very advanced keratoconus with a white cataract in the only functioning eye. This report highlights the dilemmas which were encountered and learning points on selection of the safest, yet effective management options in such patients.
\end{abstract}

Keywords: Dementia, Downs syndrome, Keratoconus.

How to cite this article: Ali $\mathrm{H}$, Nanavaty MA. Clinical Dilemmas in the Management of a Monocular Patient with Down's Syndrome, Dementia, Advanced Keratoconus, and a Mature Cataract. Int J Kerat Ect Cor Dis 2016;5(2):81-84.

\section{Source of support: Nil}

Conflict of interest: None

\section{INTRODUCTION}

There is a $66.7 \%$ prevalence of visual impairment in patients with Down's syndrome and profound intellectual disabilities over the age of 50. Visual impairment in patients with Down's syndrome has a stronger relationship with advancing age, particularly over 50 years of age. ${ }^{1,2}$ Optical rehabilitation is important in improving quality of life, particularly in those with intellectual disabilities.

The prevalence of keratoconus in patients with Down's syndrome is estimated to be between 3 and $20 \% \cdot{ }^{3-5}$ It is thought to be precipitated by vigorous eye rubbing to relieve itchiness. Patients with Down's syndrome can develop corneal hydrops, which results in a further

\footnotetext{
${ }^{1}$ Resident, ${ }^{2}$ Consultant

1,2 Sussex Eye Hospital, Brighton and Sussex University Hospitals NHS Trust, Eastern Road, Brighton, Sussex, UK

Corresponding Author: Mayank A Nanavaty, Consultant Sussex Eye Hospital, Brighton and Sussex University Hospitals NHS Trust, Eastern Road, Brighton, Sussex, UK, Phone: +7947166134, e-mail: mayank.nanavaty@bsuh.nhs.uk
}

deterioration in vision. Patients can develop central corneal opacification and in some instances corneal perforation. ${ }^{6,7}$ It is also known that a number of Down's syndrome patients will gradually develop an Alzheimerlike dementia, which will add to the complexity of their medical health. ${ }^{8}$

The diagnosis, monitoring, management, and visual rehabilitation of keratoconus can be difficult in patients with Down's syndrome, which is coexistent with dementia and poor general health. This is, particularly, so if the keratoconus is advanced in the only functioning eye with a concomitant dense white cataract. The postoperative period in patients who require surgical intervention can be especially challenging. Careful and thoughtful planning is required to prevent any complications leading to permanent loss of vision. We discuss one such case with the dilemmas encountered and learning points in helping decide the appropriate management plan in such cases.

\section{CASE REPORT}

A 60-year-old female patient with Down's syndrome living in a care home was referred to our clinic. She was thought to have visual impairment secondary to keratoconus and bilateral cataracts. She was also noted to rub her eyes regularly. The staff at her residential home had noticed a further deterioration in her vision in the past few months. She was also requiring further help with her activities of daily living. This was particularly noted as she was having problems with walking and seeing her food at meal times. The patient had difficulty with verbal communication, phobias of hospitals, and was under the dementia care pathway.

The visual acuity and slit-lamp examinations were not possible during her clinic appointment due to her uncooperative and aggressive behavior as the hospital environment disturbed her.

Options which were available to us at this stage were:

- Defer any examination under general anesthesia and/ or intervention(s)

- Attempt an examination under general anesthesia once if possible, and offer appropriate intervention at the same time.

If we deferred further assessment and/or management, it could risk her losing all sight and be detrimental 
to her overall well-being. It was, therefore, decided to administer a general anesthetic in the safest possible manner and to attempt an intervention that was in her best interest in this one and only opportunity. Giving general anesthesia was risky due to her ongoing cardiac issues, extreme difficulty in getting an intravenous access, and intubation difficulties. A multidisciplinary meeting with the Learning Disability Team, the patient's social workers, care home staff, nurses, and surgeon was arranged prior to the surgery. A formal letter of consent was obtained from the patient's elderly mother, who had power of attorney, as she could not attend the meeting prior to the surgery. This team was responsible for taking decisions on patient's best interest and welfare. Preoperative, operative, and postoperative strategies were discussed in great detail. During this process, the team already decided against splinting patient's upper limbs for 6 weeks postoperatively due to her dementia, poor general health, and risk of further aggravating the patient's behavior.

On the day of assessment, after a successful general anesthesia, the examination under the surgical microscope revealed an old perforated right cornea with iris plugging. No anterior chamber or inflammation was present (Fig. 1). Examination also revealed very advanced keratoconus in the left eye (Fig. 2A) with severe thinning of cornea and Descemet's breaks. The left eye also had a dense white cataract (Fig. 2B). B-scan ultrasonography on the table showed no evidence of retinal detachment. The left eye felt softer on digital palpation as an indicator of intraocular pressure assessment. Keratometry on the left eye was attempted with a hand held keratometer on the operating table, but due to the extreme steepness of the cornea, keratometric readings could not be obtained. Therefore an estimated average $\mathrm{K}$ reading of 55.00 Diopter was used for the intraocular lens (IOL) calculation. A manual A-scan ultrasound biometry recorded an axial length of $24 \mathrm{~mm}$ and it estimated an IOL power of 0 Diopters.

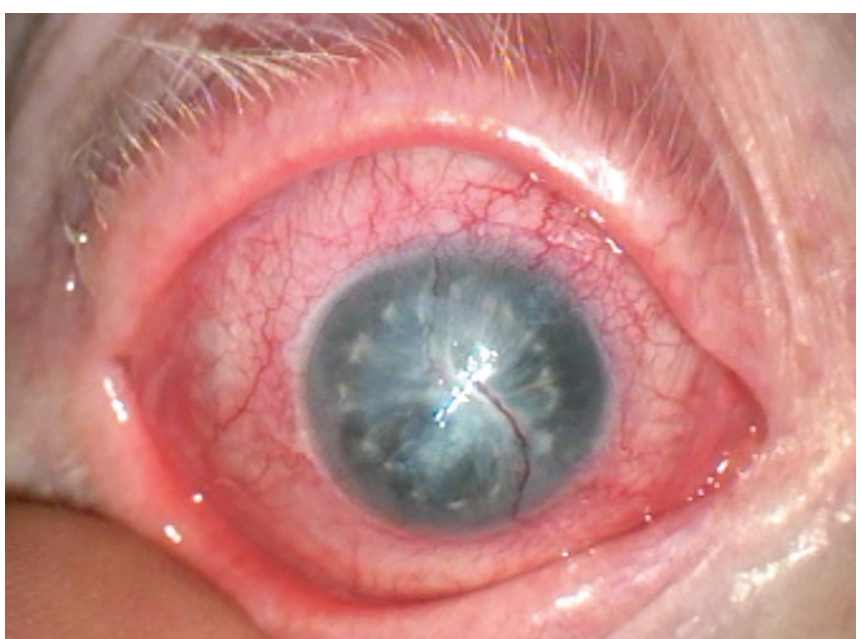

Fig. 1: Right eye with a sealed perforation of the cornea

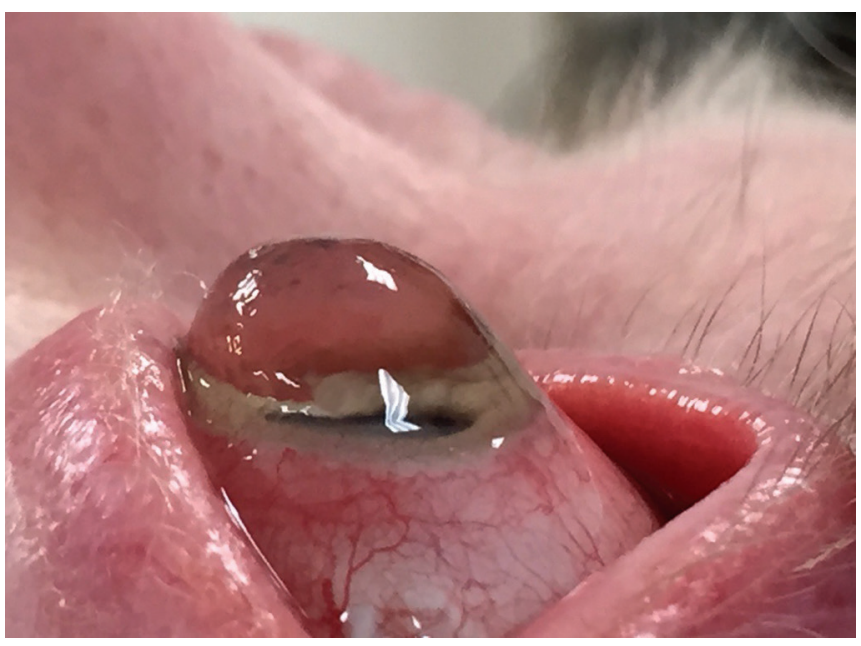

Fig. 2A: Profile of the left eye with advanced keratoconus

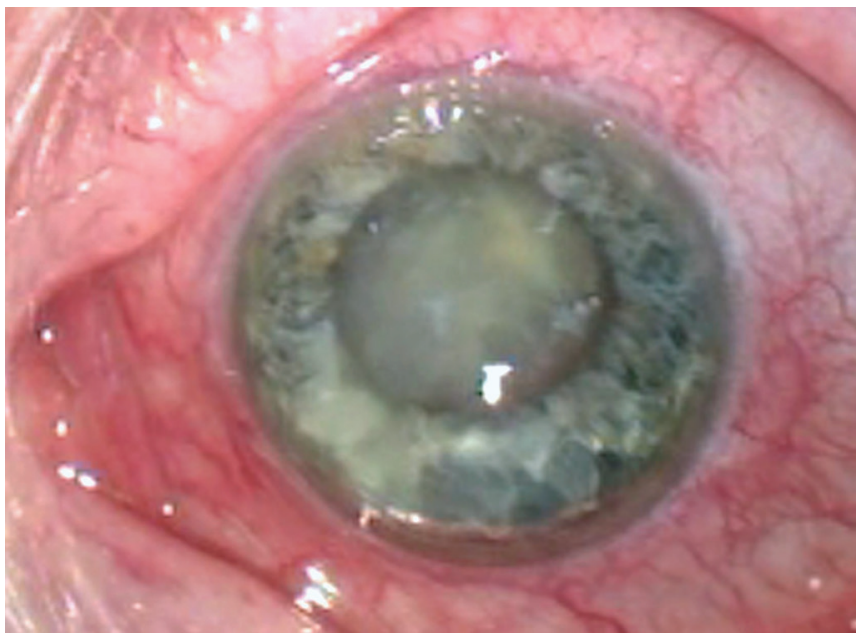

Fig. 2B: Left eye with advanced keratoconus and a mature cataract

Options which were available to us at this stage for her left eye were:

- Plan for a cataract surgery combined with a deep anterior lamellar/penetrating keratoplasty at a later date

- To proceed with cataract surgery without an IOL, as the biometry was 0 Diopters

- To proceed with cataract surgery with an IOL

- To protect the eye and the cornea from perforation postoperatively by splinting patients hands for 6 weeks postoperatively. This option was already rejected in the preassessment meeting prior to the surgery

- To protect the eye and the cornea with a lid procedure, e.g., lateral tarsorraphy or botulinum toxin injection.

As the patient was noted to have significant worsening in her vision-dependent daily activities, option 2 was chosen after deliberation. The decision was made to perform a left cataract extraction with an IOL insertion. Phacoemulsification via a superior corneal incision was performed. Vision blue was used to complete the rhexis. 


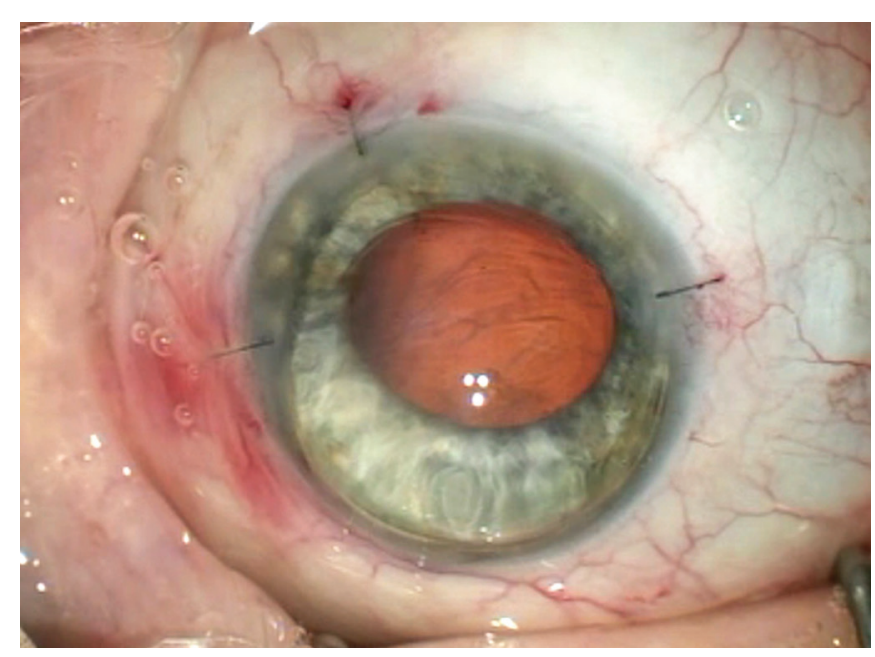

Fig. 2C: Postoperative picture of left eye with 10-0 nylon sutures on incisions

A 1.0 Diopter IOL (Rayner IOL Ltd, Hove, UK) was selected. The IOL was placed within the capsular bag. Corneal incisions were closed with 10-0 Nylon sutures and a left partial lateral tarsorrhaphy was carried out to protect the eye from accidental injury while ensuring that her visual axis was clear (Figs 2C and D). The patient was sent to her care home and a personal care taker was assigned to ensure that the patient did not touch the eye for the initial 6 postoperative weeks.

The patient was reviewed at her residential home 3 and 8 weeks after surgery as the patient refused to visit the hospital. Visual acuity and slit-lamp assessment were not possible as the patient was highly uncooperative and aggressive. On direct ophthlamoscopic examination, the lateral tarsorrhaphy was intact and the eye was white with no signs of postoperative inflammation. Her care takers had noticed a difference in her behavior. Her mobility around her surroundings had improved. She was also able to see her food.

\section{DISCUSSION}

We present the challenges and dilemmas faced when offering the most appropriate, safe, and effective management of a monocular patient with a dense white cataract and advanced keratoconus with additional comorbidities of dementia, Down's syndrome, and poor general health preventing multiple general anesthetics.

Outcomes of penetrating keratoplasties in patients with learning disabilities are significantly lower than in patients without learning disabilities. ${ }^{9-11}$ This is due to coexisting diseases, such as atopy and behavioral issues, such as persistent eye-rubbing. In our case, a decision was made not to perform a penetrating keratoplasty due to a number of issues: Concerns regarding the postoperative care of the keratoplasty; risk of blinding complications in her only eye which may not be amenable to repair due

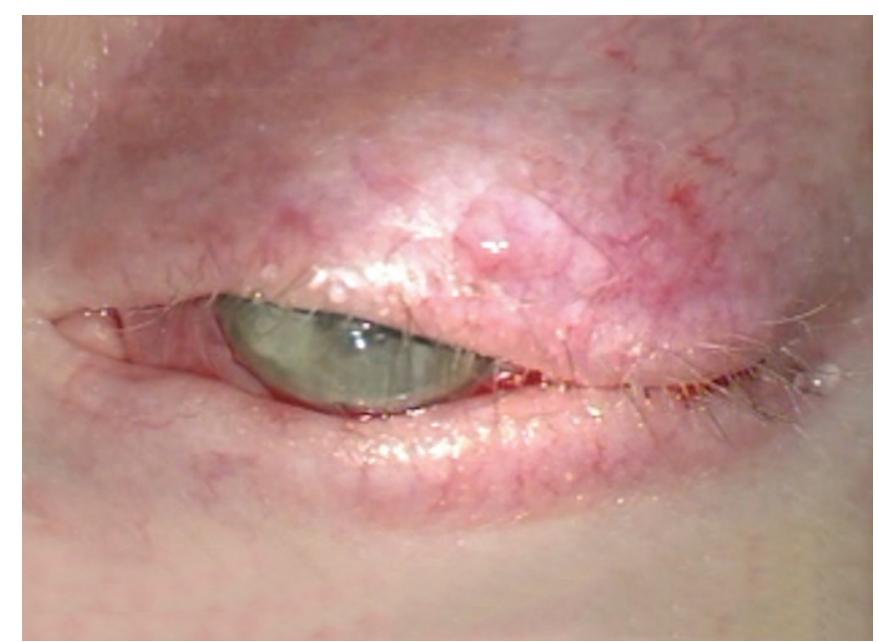

Fig. 2D: Permanent left lateral tarsorrhaphy

to life-threatening risks of multiple general anesthetic procedures; difficult intravenous access; and complex intubation due to the facial shape, thick tongue, and subglottic stenosis. ${ }^{12,13}$

The thought to leave the patient aphakic was rejected in order to prevent aphakic glaucoma, posterior capsule opacification-related issues, and retinal complications, ${ }^{14}$ as these would require further general anesthetic procedures to manage.

Corneal perforation in patients with keratoconus can be as a result of acute hydrops. There are a few reported cases of spontaneous rupture of the cornea not related to trauma in patients with keratoconus. ${ }^{15}$ There have been even fewer cases of perforations in patients with Down's Syndrome and keratoconus. ${ }^{16}$ Conservative management for corneal perforations tends to be with soft contact lenses, cycloplegics, aqueous suppressants, and pressure patching (if neither of these are possible than lid lower procedures like botolinum toxin injection or tarsorrhaphy). Penetrating keratoplasties may need to be the definitive treatment. However, a number of studies indicate that clear corneal grafts with improved postoperative visual acuities can be obtained. A retrospective study by Koller et al ${ }^{17}$ looked at the outcomes of all keratoplasties in patients with intellectual disabilities within their department. In this study $69 \%$ of the patients had a diagnosis of Down's Syndrome and 94\% of primary keratoplasties were for keratoconus. The graft survival rate of the primary keratoplasty was $86 \%$ at 27 months to 11 years follow-up (of 6 months to 20 years). At final follow-up a clear graft was present in $89 \%$ of cases. Another study looked at 21 penetrating keratoplasties performed on 18 eyes of patients with Down's Syndromes. With a mean follow-up of 34.9 months (ranging from 4 to 88 months), clear grafts were seen. Some patients had complicated follow-up periods due to broken sutures and difficulties 
with suture removal. ${ }^{18} \mathrm{~A}$ factor determining long-term graft survival, in patients with learning disabilities, is postulated to be dependent on conscientiousness of the care takers with the postoperative care.

We decided not to interfere with the right eye as the only options would be anterior segment reconstruction with cataract surgery (without or without IOL). This intervention could have led to secondary complications increasing the patients discomfort and thus affecting her general well-being. Given that the intraocular pressure was felt to be low and the appearance was suggestive of a long-standing perforation that had sealed, it was thought that the right eye was likely to become phthisi$\mathrm{cal}$ in the near future. Performing a deep anterior lamellar or penetrating keratoplasty combined with cataract surgery \pm IOL implantation was decided to be a very risky option in our patient due to the severity of her dementia and comorbidities.

It was felt that there would be a risk of wound dehiscence due to eye-rubbing in our patient. Therefore, 10-0 nylon sutures were inserted on the main and paracentesis incisions. On a longer term, it is important to reduce the risk of corneal perforation due to excessive thinning. We decided to perform a small lateral tarsorrhaphy to give some eyelid protection to the already thinned cornea. This was performed leaving the central visual axis open. Botulinum toxin injection would require multiple general anesthetic and may take a few days to be fully effective, so this was not thought to be an option for our patient. Our patient was noted to have improvement in her visual behavior at her care home, as she could see to eat and make her way around the house. She needed a personal care taker to assist her during the first 6 weeks and also to ensure that she did not touch or rub her eyes.

Our case highlights the dilemmas and gives the rationale for the selection of safest, yet effective option for reasonable visual rehabilitation for a monocular patient with Down's syndrome, dementia, and poor general heath. The risk and benefits of each of the management options should be discussed in the context to individual patients and their needs. As can often be the case, doing less can be more for such patients in the long-term.

\section{REFERENCES}

1. van Splunder J, Stilma JS, Bernsen RM, Evenhuis HM. Prevalence of ocular diagnoses found on screening 1539 adults with intellectual disabilities. Ophthalmology 2004 Aug;111(8): 1457-1463.

2. van Splunder J, Stilma JS, Bernsen RM, Evenhuis HM. Prevalence of visual impairment in adults with intellectual disabilities in the Netherlands: cross-sectional study. Eye (Lond) 2006 Sep;20(9):1004-1010.

3. García García GP, Martínez JB. Outcomes of penetrating keratoplasty in mentally retarded patients with keratoconus. Cornea 2008 Oct;27(9):980-987.

4. McElvanney AM, Adhikary HP. Penetrating keratoplasty in the mentally retarded. Eye (Lond) 1997;11(Pt 6):786-789.

5. Haugen $\mathrm{OH}$. Keratoconus in the mentally retarded. Acta Ophthalmol (Copenh) 1992 Feb;70(1):111-114.

6. Stoiber J, Muss W, Ruckhofer J, Grabner G. Acute keratoconus with perforation in a patient with Down's syndrome. Br J Ophthalmol 2003 Jan;87(1):120.

7. Fan Gaskin JC, Patel DV, McGhee CN. Acute corneal hydrops in keratoconus - new perspectives. Am J Ophthalmol 2014 May;157(5):921-928.

8. Oliver C, Holland AJ. Down's syndrome and Alzheimer's disease: a review. Psychol Med 1986 May;16(2):307-322.

9. Völker-Dieben HJ, Odenthal MT, D'Amaro J, Kruit PJ. Surgical treatment of corneal pathology in patients with Down's syndrome. J Intellect Disabil Res 1993 Apr;37(Pt 2):169-175.

10. Fasolo A, Capuzzo C, Fornea M, Franch A, Birattari F, Carito G, Cucco F, Prosdocimo G, Sala M, Delle Noci N, et al. Risk factors for graft failure after penetrating keratoplasty: 5-year follow-up from the corneal transplant epidemiological study. Cornea 2011 Dec;30(12):1328-1335.

11. Borderie VM, Sandali O, Bullet J, Gaujoux T, Touzeau O, Laroche L. Long-term results of deep anterior lamellar versus penetrating keratoplasty. Ophthalmology 2012 Feb;119(2):249-255.

12. Beilin B, Kadari A, Shapira Y, Shulman D, Davidson JT. Anaesthetic considerations in facial reconstruction for Down's syndrome. J R Soc Med 1988 Jan;81(1):23-26.

13. Miller R, Gray SD, Cotton RT, Myer CM, Netterville J. Subglottic stenosis and Down's syndrome. Am J OtolaryngoI 1990 Jul-Aug;11(4):274-277.

14. Badr IA, Hussain HM, Jabak M, Wagoner MD. Extracapsular cataract extraction with or without posterior chamber intraocular lenses in eyes with cataract and high myopia. Ophthalmology 1995 Aug;102(8):1139-1143.

15. Mostafavi D, Chu DS. Two cases of keratoconus associated with spontaneous corneal perforation. Cornea 2010 Jul;29(7):825-827.

16. Grewal S, Laibson P, Cohen E, Rapuano C. Acute hydrops in the corneal ectasias: associated factors and outcomes. Am J Ophthalmol 2000 May;129(5):702-703.

17. Koller B, Neuhann TF, Neuhann IM. Keratoplasty in patients with intellectual disability. Cornea 2014 Jan;33(1):10-13.

18. Wroblewski KJ, Mader TH, Torres MF, Parmley VC, Rotkis WM. Long-term graft survival in patients with Down syndrome after penetrating keratoplasty. Cornea 2006 Oct;25(9):1026-1028. 